\title{
Quantum critical diffusion and thermodynamics in Lifshitz holography
}

\author{
Brandon W. Langley, ${ }^{*}$ Udit M. Gupta, ${ }^{\dagger}$ and Philip W. Phillips \\ Institute of Condensed Matter Theory and Department of Physics, University of Illinois at Urbana- \\ Champaign, Urbana, Illinois 61801, USA
}

(Received 28 August 2020; accepted 4 January 2021; published 22 January 2021)

\begin{abstract}
We present the full charge and energy diffusion constants for the Einstein-Maxwell dilaton (EMD) action for Lifshitz spacetime characterized by a dynamical critical exponent $z$. Therein we compute the fully renormalized static thermodynamic potential explicitly, which confirms the forms of all thermodynamic quantities including the Bekenstein-Hawking entropy and Smarr-like relationship. All thermodynamics are based on a direct computation of the free energy. Our exact computation demonstrates a modification to the Lifshitz-Ward identity for the EMD theory. For transport, we target our analysis at finite chemical potential and include axion fields to generate momentum dissipation. While our exact results corroborate anticipated bounds, we are able to demonstrate that the diffusivities are governed by the engineering dimension of the diffusion coefficient, $[D]=2-z$. Consequently, a $\beta$ function defined as the derivative of the trace of the diffusion matrix with respect to the effective lattice spacing changes sign precisely at $z=2$. At $z=2$, the diffusion equation exhibits perfect scale invariance and the corresponding diffusion constant is the pure number $1 / d_{s}$ for both the charge and energy sectors, where $d_{s}$ is the number of spatial dimensions. Further, we find that as $z \rightarrow \infty$, the charge diffusion constant vanishes, indicating charge localization. Deviation from universal decoupled transport obtains when either the chemical potential or momentum dissipation are large relative to temperature, an echo of strong thermoelectric interactions.
\end{abstract}

DOI: 10.1103/PhysRevD.103.026016

\section{INTRODUCTION}

Because most condensed matter systems do not conform to the full Lorentz symmetry and contain dynamical behavior characterized by Lifshitz transitions [1-4], tailoring the AdS/CFT program to condensed matter systems such as the cuprates requires a considerable extension. The simplest proffer to engineer such a nonrelativistic setup is a Lifshitz geometry characterized by a dynamical critical exponent $z$ [5-9]. A metric ansatz that encodes the features of both the dynamical exponent and a finite temperature is the form

$$
d s^{2}=\frac{d r^{2}}{r^{2} f(r)}-r^{2 z} f(r) d t^{2}+r^{2} d \vec{x}_{d_{s}}^{2}
$$

which defines a horizon by the largest root of $f\left(r_{+}\right)=0$ and boundary at $r \rightarrow \infty$ where $f \rightarrow 1$. This ansatz encapsulates the scaling symmetry

\footnotetext{
*blangle2@illinois.edu †uditmg2@illinois.edu

dimer@illinois.edu
}

Published by the American Physical Society under the terms of the Creative Commons Attribution 4.0 International license. Further distribution of this work must maintain attribution to the author(s) and the published article's title, journal citation, and DOI. Funded by SCOAP.

$$
r \rightarrow c^{-1} r, \quad t \rightarrow c^{z} t, \quad x^{i} \rightarrow c x^{i}
$$

at the boundary. For $z \neq 1$, this metric ansatz cannot be a vacuum solution to the Einstein equations. Indeed, such a Lifshitz geometry requires a nontrivial bulk stress-energy tensor. Herein lies the problem: there is no unique way of engineering the requisite stress-energy tensor.

A full analytic solution to an asymptotically Lifshitz geometry that features a black hole can be constructed via an Einstein-Maxwell-dilaton (EMD) action. This model is a direct extension of the anti-de Sitter (AdS)-ReissnerNordström black hole to $z \neq 1$. This action is well known in the literature as it has served as the workhorse for most of the Lifshitz papers [10-16].

Our work addressed the open problem of the construction of the transport properties in a fully renormalized solution to the EMD action that features both a chemical potential and a set of spatially dependent axion fields that induce momentum dissipation for general $z$ and dimensionality. The action is addressed completely at the level of the static background and direct current (DC) transport. This range of analysis allows us to compute the set of both static susceptibilities and conductivities under a uniform "Lifshitz charge," which by the Einstein relations obtain the full set of thermoelectric diffusion constants. We are able to compare and contrast our results with universal features of diffusivity by Blake et al. $[17,18]$. They consider the limits of decoupled charge and 
thermal diffusion, and we find exact agreement in this limit. However, we find deviations from such behavior when matter interactions are cranked such that the thermoelectric coupling is significant.

A significant result, Fig. 1, we obtain once all the dust settles is that the length dependence of the transport properties, although they are governed by several independent scales ranging from the chemical potential to the strength of momentum dissipation, are ultimately controlled by the engineering dimension $[D]$ of the diffusion constants; by inspection of the diffusion equation, $[D]=2-z$. Consequently, the effective $\beta$ function [19],

$$
\beta \equiv \frac{\partial}{\partial \ell} \frac{(\operatorname{tr} \boldsymbol{D}) T}{v_{B}^{2}},
$$

should exhibit universal features as a function of the characteristic length scale $\ell$. In Eq. (3), we measure the diffusion matrix $\boldsymbol{D}$ against the characteristic scale $v_{B}^{2} / T$, defined in terms of the butterfly velocity and the temperature [17]. We find that $\operatorname{sgn}(\beta)=\operatorname{sgn}(2-z)$, indicating a fixed point at $z=2$. At the scale-invariant point $z=2$, diffusion is given exactly by the dimensionless number

$$
D(z=2)=\frac{1}{d_{s}} .
$$

Our diffusion constants are strictly positive unlike the previous results with restricted range of validity for $z$ $[20,21]$. We find $z=2$ corresponds to the fixed point associated with the length dependence of the diffusivities, in direct analogy with the $\beta$ function in Anderson

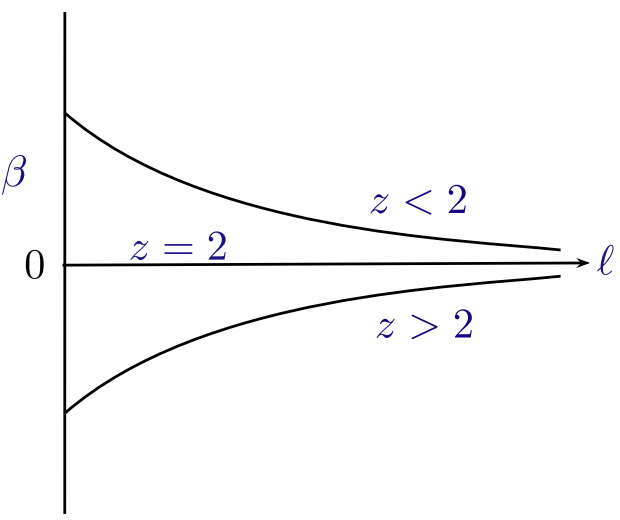

FIG. 1. Heuristic plot of the $\beta$ function defined as the derivative of the trace of the diffusion matrix with respect to the system size. At $z=2$, the diffusion coefficient is a universal dimensionless constant determined solely by the number of spatial dimensions. Away from $z=2$, the diffusion constants (either charge or energy) have opposite slopes relative to an increase in the system size indicating a fixed point at $z=2$. Regardless of $z, \beta$ asymptotes to zero as the system size increases, indicating the bounded nature of the diffusivities. At $z=2$, the diffusion equation is scale invariant. localization [19]. Our conclusion here is made possible entirely because we have a regularizable boundary theory.

We find in general that diffusivity bounds $[22,23]$ do indeed exist for Lifshitz holography, with two possible violations. The first for $z=1$, as is standard, has a divergent energy diffusion constant in the absence of momentum dissipation, caused by the inability of any sourced momentum to relax. The second occurs at $z \rightarrow \infty$, whereupon charge becomes completely localized and does not diffuse, manifest in the vanishing of the upper bound in the respective diffusion constant.

As a final important note, our exact treatment finds that the Ward identity associated with the Lifshitz symmetry in the EMD model. We find that the boundary stress-energy tensor $\mathbb{T}_{b}^{a}$ and dilaton response $\lambda_{\phi} \mathbb{O}_{\phi}$ obey

$$
z \mathbb{W}^{t}+\mathbb{W}^{x^{i}}{ }_{x^{i}}+\lambda_{\phi} \mathbb{O}_{\phi}=0
$$

which aligns with the encoding of the Lifshitz symmetry via dilatation. This is a slight contrast to the usual identity which omits any contribution from the dilaton. In spite of this, these results are not contradictory in the context of $[14,15]$ for instance, due to an alternative construction of the boundary theory and the dual stress-energy tensor. In fact, due to the nebulous nature of the interpretation of the boundary geometry, there is some leeway in the formulation.

\section{AN ABRIDGED HISTORY OF LIFSHITZ HOLOGRAPHY}

The short history of Lifshitz holography is one of many fits and starts. The initial advance [5] in this context supplemented the standard bulk Lagrangian with two gauge fields, a 1-form and a 2-form both, coupled together via a topological term that controls the dynamical exponent $z$. Though a clean analytic construction, it is restricted to $d_{s}=2$ spatial dimensions and is not amenable to emblackening factors which would encode a horizon.

Several other models have been proposed and analyzed [24], such as the Einstein-Proca model [25,26]. However the EMD model imbibes the most robust features for thermodynamics. One of the long-standing issues with this theory is the absence of a renormalization scheme for the boundary action, unlike AdS [27]. Without such a scheme, there is no real interpretation of the response functions and thermodynamics of the system. While some models could be worked out under specific conditions-the $z=2$ Schrödinger symmetry [28] a case in point —or certain response functions obtained such as the specific heat [29], the general theory remained elusive [30]. One of the culprits is the $U(1)$ field responsible for turning on $z \neq 1$ is poorly behaved at the boundary. There have been several proposals for dealing with this divergence. The original proposition for handling this divergence was to perform a Legendre transformation [13] to instead consider 
the stable dual $U(1)$ current as the fundamental variational instead of the electric field.

The Legendre transformation alone leaves the scheme incomplete. An alternative was proposed. Kiritsis and Matsuo's hydrodynamic ansatz, wherein the constant parameters controlling the static solution are promoted to slowly varying functions of time and space, permits an analytic solution to the induced fluctuation equations. As its title suggests, this formulation allows all thermodynamic quantities to be expressed as components of a nonrelativistic fluid. The controlled expansion allows them to make contact with a renormalization scheme without performing a Legendre transformation. Their solution involves an infinite series of counterterms involving the divergent $U(1)$ field and supports slowly varying transport properties.

The next stride was made by Cremonini et al. who examined the transverse modes of the EMD theory [31,32]. Previous attempts on this model ignored the crucial coupling between the two $U(1)$ fields [33,34], which must be present for a nontrivial solution. Cremonini et al. sought heat and charge transport response functions of the system at low frequencies. Therein, their program enabled a renormalization of specifically the transverse modes: they (1) perform the Legendre transformation for the divergent $U(1)$ field, (2) build a second Arnowitt-Deser-Misner (ADM) breakdown to separate time and space in the boundary with a timelike shift, and (3) renormalize the theory in terms of the corresponding $U(1)$ current and timelike shift. A feature of this scheme is that the counterterms actually depend on both the non-normalizable and normalizable modes of the model. A renormalization scheme depending upon the theory's renormalizable modes is usually problematic, but in fact the counterterms can be state dependent for systems perturbed by irrelevant operators [35].

\section{ACTION AND STATIC BACKGROUND}

We suppose the EMD action

$$
I=-\int_{\mathcal{M}} \sqrt{-g}\left(R-\frac{1}{2}(\nabla \phi)^{2}-V(\phi)-\frac{1}{4} \sum_{q=1}^{2} Z_{q}(\phi) F_{q}^{2}-\frac{1}{2} X(\phi) \sum_{I=1}^{d_{s}}\left(\nabla \chi_{I}\right)^{2}\right)-\int_{\partial \mathcal{M}} \sqrt{-\gamma} 2 K+I_{\text {c.t. }},
$$

where $I_{\text {c.t. }}$ is a smattering of counterterms to give us welldefined boundary action. Here, $\mathcal{M}$ is a $d_{s}+2$-dimensional Lorentzian manifold and $\partial \mathcal{M}$ is its boundary. This action features two $U(1)$ fields, one which will serve to assist turning on a nontrivial $z \neq 1$ solution and one which generates a standard chemical potential $\mu$, a straight extension of the usual AdS-Reissner-Nordström black hole. The axion fields will generate momentum dissipation. This action yields the equations of motion

$$
\begin{aligned}
E_{a b}= & R_{a b}-\frac{1}{2} \nabla_{a} \phi \nabla_{b} \phi-\frac{1}{d_{s}} V(\phi) g_{a b} \\
& -\frac{1}{2} \sum_{q=1}^{2} Z_{q}(\phi)\left(F_{q, a c} F_{q, b}{ }^{c}-\frac{1}{2 d_{s}} F_{q}^{2} g_{a b}\right) \\
& -\frac{1}{2} X(\phi) \sum_{i=1}^{d_{s}} \nabla_{a} \chi_{i} \nabla_{b} \chi_{I}=0, \\
D_{\phi}= & \square \phi-V^{\prime}(\phi)-\frac{1}{4} \sum_{q=1}^{2} Z_{q}^{\prime}(\phi) F_{q}^{2}-\frac{1}{2} X^{\prime}(\phi) \sum_{I=1}^{d_{s}}\left(\nabla_{I}\right)^{2} \\
= & 0, \\
M_{q}^{a}= & \nabla_{b}\left(Z_{q}(\phi) F_{q}^{a b}\right)=0, \\
\Xi_{I}= & \nabla_{a}\left(X(\phi) \nabla^{a} \chi_{I}\right)=0 .
\end{aligned}
$$

This system has a static solution, with matter fields given by

$$
\begin{aligned}
\phi & =\lambda_{\phi}\left(\ln r+\phi_{1}\right), \quad \lambda_{\phi}=\sqrt{2 d_{s}(z-1)}, \\
V(\phi) & =-\left(z+d_{s}-1\right)\left(z+d_{s}\right), \\
Z_{q}(\phi) & =e^{2 \frac{\lambda_{q}}{\lambda_{\phi}} \phi}, \quad X(\phi)=e^{2 \frac{x_{\phi}}{\lambda_{\phi}} \phi}, \\
A_{1} & =\sqrt{\frac{2(z-1)}{z+d_{s}}} e^{d_{s} \phi_{1}}\left(r^{z+d_{s}}-r_{+}^{z+d_{s}}\right) d t, \quad \lambda_{1}=-d_{s}, \\
A_{2} & =\mu_{2}\left[1-\left(\frac{r_{+}}{r}\right)^{z+d_{s}-2}\right] d t, \quad \lambda_{2}=z-1, \\
\chi_{I} & =k \delta_{I i} x^{i}, \quad \lambda_{\chi}=-(z-1),
\end{aligned}
$$

and the emblackening factor

$$
\begin{aligned}
f(r)=1 & +\frac{z+d_{s}-2}{2 d_{s}} \frac{\mu_{2}^{2} e^{2(z-1) \phi_{1}}}{r_{+}^{2}}\left(\frac{r_{+}}{r}\right)^{2\left(z+d_{s}-1\right)} \\
& +\frac{1}{2\left(z-d_{s}\right)} \frac{k^{2} e^{-2(z-1) \phi_{1}}}{r_{+}^{2 z}}\left(\frac{r_{+}}{r}\right)^{2 z}-M\left(\frac{r_{+}}{r}\right)^{z+d_{s}},
\end{aligned}
$$

where $M$ is the mass of the black hole,

$M=1+\frac{z+d_{s}-2}{2 d_{s}} \frac{\mu_{2}^{2} e^{2(z-1) \phi_{1}}}{r_{+}^{2}}+\frac{1}{2\left(z-d_{s}\right)} \frac{k^{2} e^{-2(z-1) \phi_{1}}}{r_{+}^{2 z}}$. 
Note that this solution demands $z \geq 1$. For the critical case $d_{s}=z$, a logarithmic singularity obtains, and the emblackening factor takes the form

$$
\begin{aligned}
f(r)= & 1+\frac{z-1}{z} \frac{\mu_{2}^{2} e^{2(z-1) \phi_{1}}}{r_{+}^{2}}\left(\frac{r_{+}}{r}\right)^{4 z-2} \\
& -\left[1+\frac{z-1}{z} \frac{\mu_{2}^{2} e^{2(z-1) \phi_{1}}}{r_{+}^{2}}-\frac{1}{2} \frac{k^{2} e^{-2(z-1) \phi_{1}}}{r_{+}^{2 z}} \ln \left(\frac{r_{+}}{r}\right)\right] \\
& \times\left(\frac{r_{+}}{r}\right)^{2 z} .
\end{aligned}
$$

For the analysis in this paper, we will avoid this singularity and forbid $k \neq 0$ for this critical case, as it inherently would not modify the thermodynamics after renormalization. The temperature is

$$
\begin{aligned}
T & =\frac{r_{+}^{z+1} f^{\prime}\left(r_{+}\right)}{4 \pi} \\
& =\frac{r_{+}^{z}}{4 \pi}\left(z+d_{s}-\frac{\left(z+d_{s}-2\right)^{2}}{2 d_{s}} \frac{\mu_{2}^{2} e^{2(z-1) \phi_{1}}}{r_{+}^{2}}-\frac{1}{2} \frac{k^{2} e^{-2(z-1) \phi_{1}}}{r_{+}^{2 z}}\right),
\end{aligned}
$$

derived by eliminating the conical singularity at the horizon.

\section{RENORMALIZATION}

In order to have a well-defined boundary action, and therefore dictionary, we must have a renormalization scheme to ensure all divergences are removed.

As the divergent terms can be arranged in a power series that necessarily terminates, for finite $z$ and $d_{s}$, any divergence can be balanced by a finite number of counterterms. If our action's variation can be expressed as

$$
\delta I=\int_{\partial \mathcal{M}} \sum_{n} \Pi_{n}^{(\phi)} \delta \phi_{n}
$$

where we have symplectic data and the products of all the variations $\delta \phi_{n}$ and their radial conjugate momenta $\Pi_{n}^{(\phi)}$ are $O(1)$ as $r \rightarrow \infty$, then we have ascertained a renormalized boundary action. This scheme can be implemented not requiring a full, generalized solution but rather utilizing details of specific solutions only as necessary. The scheme for the low-frequency transverse transport properties was first laid out in [32]. If the reader seeks a more generalized approach beyond the scope of specific solutions, it is wise to turn to the radial Hamilton-Jacobi equations to define a boundary potential via a functional derivative expansion, as in [36,37]. For our purposes, we need only examine the possible forms of nontrivial counterterms to ascertain the renormalized action within the scope of our solution.
The variation of the action (6) yields

$$
\begin{aligned}
\delta I & =\int_{\partial \mathcal{M}}\left(\frac{1}{2} T^{a b} \delta \gamma_{a b}-\sum_{q=1}^{2} J_{q}^{a} \delta A_{q, a}+\mathcal{O}_{\phi} \delta \phi\right), \\
T^{a b} & =2 \sqrt{-\gamma}\left(K^{a b}-K \gamma^{a b}\right), \quad J_{q}^{a}=\sqrt{-\gamma} N_{b} Z_{q}(\phi) F_{q}^{a b}, \\
\mathcal{O}_{\phi} & =\sqrt{-\gamma} N_{a} \nabla^{a} \phi,
\end{aligned}
$$

where $N^{a}$ is a unit vector normal to the boundary hypersurface foliating the bulk spacetime along the radial direction, and $K_{a b} \equiv \nabla_{(a} N_{b)}$. We have neglected the conjugate momenta for the axions as they vanish at the level of the static background and will not contribute to the DC currents. We absolutely do not have renormalized symplectic data, with the most glaring issue being that $A_{1}$ is divergent. However, its conjugate momentum is $O(1)$; hence we can switch to a stable scheme via a Legendre transformation. We select the counterterms

$$
I_{\text {c.t. }}=\int_{\partial \mathcal{M}}\left(A_{1, a} J_{1}^{a}+c_{0}^{(\mathrm{vol})} \sqrt{-\gamma}+c_{0}^{\left(J_{1}^{2}\right)} \frac{J_{1}^{2}}{\sqrt{-\gamma} Z_{1}(\phi)}+\cdots\right),
$$

where we can see the first term switches our potential to vary under $J_{1}$ instead of $A_{1}$, changing the boundary condition from Dirichlet to Neumann, as proposed in [13]. We will thusly refer to this action as $I_{N}$. While $A_{2}$ and $J_{2}$ already combine to yield an $O(1)$ boundary contribution, we must yet ensure the other responses are renormalized. Note that $J_{1}^{2}$ counterterm is necessary for a finite response but it can also take the interpretation of a double-trace deformation. Equipped with these counterterms, we define the new responses as

$$
\begin{aligned}
\mathbb{T}_{b}^{a}= & 2 \sqrt{-\gamma}\left(K_{b}^{a}-K \delta_{b}^{a}+\frac{1}{2} c_{0}^{(\mathrm{vol})} \delta^{a}{ }_{b}\right) \\
& +\frac{2 c_{0}^{\left(J_{1}^{2}\right)}}{\sqrt{-\gamma} Z_{1}(\phi)}\left(J_{1}^{a} J_{1, b}-\frac{1}{2} J_{1}^{2} \delta^{a}{ }_{b}\right)+\cdots, \\
\mathbb{A}_{1, a}= & A_{1, a}+\frac{2 c_{0}^{\left(J_{1}^{2}\right)} J_{1, a}}{\sqrt{-\gamma} Z_{1}(\phi)}+\cdots, \\
\mathbb{O}_{\phi}= & \sqrt{-\gamma} N_{a} \nabla^{a} \phi-c_{0}^{\left(J_{1}^{2}\right)} \frac{Z_{1}^{\prime}(\phi)}{Z_{1}(\phi)^{2}} \frac{J_{1}^{2}}{\sqrt{-\gamma}}+\cdots .
\end{aligned}
$$

We find that

$$
c_{0}^{(\mathrm{vol})}=z+2 d_{s}-1, \quad c_{0}^{\left(J_{1}^{2}\right)}=\frac{1}{2\left(z+d_{s}\right)}
$$

is sufficient. These counterterms are in agreement with those used to analyze DC conductivities in [32]. Note that 
implicit in the ellipses are remaining counterterms, but the outlined contributions are the only terms that contribute to the finite action. All other terms will simply cancel divergences without contributing to the free energy and are worked out accordingly in Appendix A.

If we substitute in our static solution, we will find as $r \rightarrow \infty$,

$$
\begin{aligned}
\mathbb{T}^{t}{ }_{t} & =d_{s} M r_{+}^{z+d_{s}}+\cdots, \\
\mathbb{T}^{x^{i}}{ }_{x^{j}} & =-M r_{+}^{z+d_{s}} \delta^{i}{ }_{j}+\cdots, \\
\mathbb{A}_{1, t} & =\sqrt{\frac{2(z-1)}{z+d_{s}}} e^{d_{s} \phi_{1}}\left(\frac{M}{2}-1\right) r_{+}^{z+d_{s}}+\cdots, \\
\mathbb{O}_{\phi} & =-\sqrt{2 d_{s}(z-1)} \frac{M}{2} r_{+}^{z+d_{s}}+\cdots .
\end{aligned}
$$

Also note that the stress-energy tensor is actually traceless, which is a bit surprising for a Lifshitz theory as this is usually indicative of a scale-invariant theory, though it is not a necessary condition. Regardless, the Lifshitz scaling symmetry is manifestly encoded as a dilatation. This is reflected in the new Ward identity,

$$
z \mathbb{W}_{t}^{t}+T^{x^{i}}{ }_{x^{i}}+\lambda_{\phi} \mathbb{O}_{\phi}=0 .
$$

This modification to the Ward identity for the anisotropic Weyl transformation [38] is perhaps not so strange. Unlike the pure AdS case, there is no simple interpretation of the boundary metric. Under separate constructions such as the Newton-Cartan background, modifications are expected [39].

While this renormalization machinery is sufficient for the static background, when it comes to the currents we will find that $z \neq 1$ causes the scaling of the source terms to be divergent. We can remove the source terms from our boundary action with a simple reformulation of our intrinsic metric,

$$
\gamma_{a b} d x^{a} d x^{b}=-n^{2}\left(d t-\frac{n_{i}}{n^{2}} d x^{i}\right)^{2}+\sigma_{i j} d x^{i} d x^{j},
$$

which functions akin to an inverted ADM formalism, which we further discuss in Appendix C.

\section{FREE ENERGY}

The free energy can be computed from the on-shell action. The Ricci scalar

$$
\begin{aligned}
R= & \frac{1}{2}(\nabla \phi)^{2}+\left(1+\frac{2}{d_{s}}\right) V(\phi) \\
& +\left(\frac{1}{4}-\frac{1}{2 d_{s}}\right) \sum_{q=1}^{2} Z_{q}(\phi) F_{q}^{2}+\frac{1}{2} X(\phi) \sum_{I=1}^{d}\left(\nabla \chi_{I}\right)^{2}
\end{aligned}
$$

can be plugged in to yield the on-shell bulk action

$$
I_{\text {bulk }}^{(\text {o.s. })}=-\int_{\mathcal{M}} \sqrt{-g}\left(\frac{2}{d_{s}} V(\phi)-\frac{1}{2 d_{s}} \sum_{q=1}^{2} Z_{q}(\phi) F_{q}^{2}\right),
$$

which we can expressly integrate. Combining with our boundary terms, we find that the full on-shell boundary action is

$$
\begin{aligned}
I_{N}^{(\text {o.s. })}= & \frac{W_{N}}{T} \\
= & \operatorname{vol}_{d_{s}} \frac{r_{+}^{z+d_{s}}}{T}\left(-z+\frac{(z-2)\left(z+d_{s}-2\right)}{2 d_{s}} \frac{\mu_{2}^{2} e^{2(z-1) \phi_{1}}}{r_{+}^{2}}\right. \\
& \left.+\frac{z}{2\left(z-d_{s}\right)} \frac{k^{2} e^{-2(z-1) \phi_{1}}}{r_{+}^{2 z}}\right),
\end{aligned}
$$

where $\operatorname{vol}_{d_{s}}$ is a $d_{s}$-dimensional spatial volume and $W_{N}$ is the "Neumann" free energy. We are working in an ensemble with $W_{N}\left(T, \phi_{1}, \mu_{2}, k\right)$, indicating our independent variables, where $r_{+}\left(T, \phi_{1}, \mu_{2}, k\right)$ is an implicit function that solves Eq. (12). A priori, we notice that the charge density $J_{1}^{t}=\sqrt{2(z-1)\left(z+d_{s}\right)} e^{-d \phi_{1}}$ is a function of only $\phi_{1}$, meaning that the parameter $\phi_{1}$ directly and singlehandedly sources both the responses $\mu_{1}$ and $\mathbb{O}_{\phi}$.

Let us compute (and verify) our thermodynamic quantities. In differential form the first law of thermodynamics is manifest,

$$
\begin{aligned}
d W_{N}= & -\mathcal{S} d T-\mathcal{Q}_{2} d \mu_{2}+\left(\mu_{1} \frac{\partial \mathcal{Q}_{1}}{\partial \phi_{1}}+\operatorname{vol}_{d_{s}} \lambda_{\phi} \mathbb{O}_{\phi}\right) d \phi_{1} \\
& -d_{s} \mathcal{O}_{k} d k
\end{aligned}
$$

where the entropy $\mathcal{S}$, charges $\mathcal{Q}_{1}$ and $\mathcal{Q}_{2}$, and dual operator $\mathcal{O}_{k}$ to the impurity $k$ are given by

$$
\begin{aligned}
\mathcal{S} & =\operatorname{vol}_{d_{s}} 4 \pi r_{+}^{d_{s}} \\
\mathcal{Q}_{1} & =\operatorname{vol}_{d_{s}} \sqrt{2(z-1)\left(z+d_{s}\right)} e^{-d_{s} \phi_{1}}=\operatorname{vol}_{d_{s}} J_{1}^{t}, \\
\mathcal{Q}_{2} & =\operatorname{vol}_{d_{s}}\left(z+d_{s}-2\right) \mu e^{2(z-1) \phi_{1}} r_{+}^{z+d_{s}-2}=\operatorname{vol}_{d_{s}} J_{2}^{t}, \\
\mathcal{O}_{k} & \equiv-\operatorname{vol}_{d_{s}} \frac{k e^{-2(z-1) \phi_{1}} r_{+}^{-z+d_{s}}}{z-d_{s}}
\end{aligned}
$$

Notably, $\operatorname{vol}_{d_{s}} r_{+}^{d_{s}}$ is exactly the surface area of the black hole and thus $\mathcal{S}$ obeys the celebrated Bekenstein-Hawking relation (where we have chosen $G_{N}=\frac{1}{16 \pi}$ ). The operator $\mathcal{O}_{k}$ functions analogously to a magnetization in response to an applied magnetic field [40]. The $1 / d_{s}$ factor is chosen to normalize the response to one spatial coordinate.

From here, it is possible to compute the system's internal energy, 


$$
\begin{aligned}
\mathcal{E} & =W_{N}+T \mathcal{S}+\mu_{2} \mathcal{Q}_{2}, \\
& =\operatorname{vol}_{d_{s}} d_{s} M r_{+}^{z+d_{s}}=\operatorname{vol}_{d_{s}} \mathbb{T}^{t},
\end{aligned}
$$

which again is self-consistent: the energy contained is proportional to the black hole's mass, and given by the $t t$ component of the stress-energy tensor.

Finally, we compute the pressure $\mathfrak{p}$. Our system's trivial volume dependence means the pressure is just the negated thermodynamical potential density in the grand canonical ensemble - namely the density of the "Dirichlet" free energy $W_{D}=W_{N}-\mu_{1} \mathcal{Q}_{1}$ - and is given by

$$
\begin{aligned}
p & =-\frac{W_{D}}{\operatorname{vol}_{d_{s}}}, \\
& =\left(M-\frac{1}{z-d_{s}} \frac{k^{2} e^{-2(z-1) \phi_{1}}}{r_{+}^{2 z}}\right) r_{+}^{z+d_{s}} \\
& =-\mathbb{T}^{x^{1}}{ }_{x^{1}}+\frac{k \mathcal{O}_{k}}{\operatorname{vol}_{d_{s}}} .
\end{aligned}
$$

As expected, turning on impurities creates the disparity $\mathfrak{p} \neq-\mathbb{T}^{x^{1}}{ }_{x^{1}}$. The simple form of the pressure guarantees the satisfaction of a Smarr-like relation,

$$
\epsilon+\mathfrak{p}=T s+\sum_{q=1}^{2} \mu_{q} \rho_{q},
$$

where $\epsilon, s$, and $\rho_{q}$ are the energy, entropy, and charge densities respectively.

\section{DC CONDUCTIVITIES}

When we consider fluctuations of the bulk spacetime and fields at the level of slowly varying gradients, the equations of motion decouple into three separate modes. A so-called sound mode from which susceptibilities can be derived, a tensor mode which expresses vorticity of the fluid, and a vector mode which contains the system's heat and charge currents $[14,15]$. To acquire the DC conductivities, we can supply linear time sources for the vector mode and extract the current responses. We take the ansatz,

$$
\begin{aligned}
\delta d s^{2} & =2 r^{2} \delta g_{r x^{1}} d r d x^{1}+2\left(-\frac{\nabla T}{T} r^{2 z} f t+r^{2} \delta g_{t x^{1}}\right) d t d x^{1} \\
\delta A_{q} & =\left(-\nabla \mu_{q} t+\frac{\nabla T}{T} A_{q, t} t+\delta A_{q, x^{1}}\right) d x^{1} \\
\delta \chi_{I} & =\delta_{I 1} \delta \chi_{1}
\end{aligned}
$$

where the perturbations-without loss of generality due to rotational symmetry-are sourced along the $x^{1}$ direction. The equations of motion come in two batches,

$$
\begin{aligned}
& -f r^{z-d_{s}+1}\left(r^{-z+d_{s}+3} \delta g_{t x^{1}}^{\prime}+\sum_{q=1}^{2} \rho_{q} \delta A_{q, x^{1}}\right)^{\prime} \\
& +k^{2} X(\phi) \delta g_{t x^{1}}=0, \\
& j^{\left(\rho_{q}\right) \prime}=0, \\
& j^{\left(\rho_{q}\right)}=-r^{z+d_{s}-1} f Z_{q}(\phi) \delta A_{q, x^{1}}^{\prime}-\rho_{q} \delta g_{t x^{1}},
\end{aligned}
$$

which are $E_{t x^{1}}$ and $M_{q}^{x^{1}}$, and

$$
\begin{aligned}
& {\left[r_{+}^{z+d_{s}+1} f^{\prime}\left(r_{+}\right)-\frac{1}{z-d_{s}} k^{2} X(\phi) r^{2(z-1)}\left(r^{-z+d_{s}}-r_{+}^{-z+d_{s}}\right)\right] \frac{\nabla T}{T}} \\
& +\sum_{q=1}^{2} \rho_{q} \nabla \mu_{q}-k X(\phi) r^{z+d_{s}+1} f\left(\delta \chi_{1}^{\prime}-k \delta g_{r x^{1}}\right)=0 \\
& -k \frac{\nabla T}{T}+r^{z-d_{s}+1}\left[r^{-z+d_{s}+3} f\left(\delta \chi_{1}^{\prime}-k \delta g_{r x^{1}}\right)\right]^{\prime}=0
\end{aligned}
$$

which are $E_{r x^{1}}$ and $\Xi_{1}$, respectively. We can see $\Xi_{1}=0$ follows from $E_{t x^{1}}=0$. Thus, $E_{r x^{1}}$ completely decouples from the other equations and acts as a first-order constraint.

The electric currents $j^{\left(\rho_{q}\right)}=J_{q}^{x^{1}}$ are conserved in the bulk, but we can construct another conserved current by considering a Killing vector as shown in Appendix B. The result is

$j^{(\mathfrak{q})}=r^{3 z+d_{s}-1} f^{2}\left(r^{-2(z-1)} f^{-1} \delta g_{t x^{1}}\right)-\sum_{q=1}^{2} A_{q, t} j^{\left(\rho_{q}\right)}$

$j^{(\mathfrak{q}) \prime}=0$,

where and the conserved bulk quantity $j^{(\mathfrak{q})}$ is the boundary heat current. We demand these functions are regular at the horizon in ingoing Eddington-Finkelstein coordinates, given by the transformation

$$
d t_{+}=d t+\frac{d r}{r^{z+1} f}
$$

and thus near the horizon we obtain $t=t_{+}-\frac{1}{4 \pi T} \ln \left(r-r_{+}\right)$. Regularity yields the asymptotic relations

$$
\begin{aligned}
\delta g_{r x^{1}} & \sim \frac{1}{k^{2} X\left(\phi_{+}\right) s T\left(r-r_{+}\right)}\left(s \nabla T+\sum_{q=1} \rho_{q} \nabla \mu_{q}\right), \\
\delta g_{t x^{1}} & \sim \frac{4 \pi}{k^{2} X\left(\phi_{+}\right) s}\left(s \nabla T+\sum_{q=1} \rho_{q} \nabla \mu_{q}\right), \\
\delta A_{q, x^{1}} & \sim \frac{1}{4 \pi T} \nabla \mu_{q} \ln \left(r-r_{+}\right),
\end{aligned}
$$

where we express our quantities in terms of the entropy and charge densities and defined $\phi_{+}=\phi\left(r_{+}\right)$. Plugging into our currents, which are conserved in the bulk, we find 


$$
\begin{aligned}
j^{(\mathfrak{q})}= & -\frac{4 \pi T}{k^{2} X\left(\phi_{+}\right)}\left(s \nabla T+\sum_{q=1}^{2} \rho_{q} \nabla \mu_{q}\right), \\
j^{\left(\rho_{q}\right)}= & -r_{+}^{d_{s}-2} Z_{q}\left(\phi_{+}\right) \nabla \mu_{q} \\
& -\frac{4 \pi \rho_{q}}{k^{2} X\left(\phi_{+}\right) s}\left(s \nabla T+\sum_{p=1}^{2} \rho_{p} \nabla \mu_{p}\right) .
\end{aligned}
$$

The heat current is related to the energy current via

$$
j^{(\mathfrak{q})}=j^{(\epsilon)}-\sum_{q=1}^{2} \mu_{q} j^{\left(\rho_{q}\right)},
$$

which of course is a measure of energy flow in excess of the energy due to charge transfer. Thus, the energy current is given by

$$
\begin{aligned}
j^{(\epsilon)}= & -r_{+}^{d_{s}-2} \sum_{q=1}^{2} Z_{q}\left(\phi_{+}\right) \mu_{q} \nabla \mu_{q} \\
& -\frac{4 \pi\left(s T+\sum_{q=1}^{2} \mu_{q} \rho_{q}\right)}{k^{2} X\left(\phi_{+}\right) s}\left(s \nabla T+\sum_{p=1}^{2} \rho_{p} \nabla \mu_{p}\right) .
\end{aligned}
$$

\section{ENERGY AND CHARGE DIFFUSION}

Let us consider the diffusion of energy and charge in our system. From hereon we can simply work with densities. The energy and charges follow continuity equations,

$$
\partial_{t} \epsilon+\nabla \cdot j^{(\epsilon)}=0, \quad \partial_{t} \rho_{q}+\nabla \cdot j^{\left(\rho_{q}\right)}=0 .
$$

In the Neumann ensemble, gradients of $T, \phi_{1}$ and $\mu_{2}$ source gradients of energy and charge density. We will examine the diffusion of the system's energy and electric charge under the constraint where the "Lifshitz charge" is completely fixed and uniform; that is, $j^{\left(\rho_{1}\right)}=0$ and $\nabla \rho_{1}=0$. Under this constraint,

$$
\begin{aligned}
\nabla \epsilon & =\left(c_{\mu_{2}}+\mu_{2} \zeta\right) \nabla T+\left(T \zeta+\mu_{2} \chi\right) \nabla \mu_{2}, \\
\nabla \rho_{2} & =\zeta \nabla T+\chi \nabla \mu_{2},
\end{aligned}
$$

where

$$
\begin{gathered}
c_{\mu_{2}}=\left.T \frac{\partial s}{\partial T}\right|_{\rho_{1}, \mu_{2}}=-T \frac{\partial^{2} w_{N}}{\partial T^{2}}, \\
\zeta=\left.\frac{\partial s}{\partial \mu_{2}}\right|_{T, \rho_{1}}=\left.\frac{\partial \rho_{2}}{\partial T}\right|_{\rho_{1}, \mu_{2}}=-\frac{\partial^{2} w_{N}}{\partial T \partial \mu_{2}}, \\
\chi=\left.\frac{\partial \rho_{2}}{\partial \mu_{2}}\right|_{T, \rho_{1}}=-\frac{\partial^{2} w_{N}}{\partial \mu_{2}^{2}}
\end{gathered}
$$

the susceptibilities are computable as second-order derivatives of the free energy density $w_{N}$.

The associated heat and charge currents,

$j^{(\mathfrak{q})}=-\bar{\kappa} \nabla T-T \alpha \nabla \mu_{2}, \quad j^{\left(\rho_{2}\right)}=-\alpha \nabla T-\sigma \nabla \mu_{2}$,

$j^{(\epsilon)}=-\left(\bar{\kappa}+\mu_{2} \alpha\right) \nabla T-\left(T \alpha+\mu_{2} \sigma\right) \nabla \mu_{2}$,

are given by, utilizing Eq. (36) and the constraint $j^{\left(\rho_{1}\right)}=0$,

$$
\begin{aligned}
\bar{\kappa} & =\frac{4 \pi s T}{\Sigma_{1} k^{2} X\left(\phi_{+}\right)}, \\
\alpha & =\frac{4 \pi \rho_{2}}{\Sigma_{1} k^{2} X\left(\phi_{+}\right)}, \\
\sigma & =r_{+}^{d_{s}-2} Z_{2}\left(\phi_{+}\right)+\frac{4 \pi \rho_{2}^{2}}{\Sigma_{1} k^{2} X\left(\phi_{+}\right) s}, \\
\Sigma_{1} & =1+\frac{\rho_{1}^{2}}{k^{2} X\left(\phi_{+}\right) Z_{1}\left(\phi_{+}\right) r_{+}^{2 d_{s}-2}},
\end{aligned}
$$

where $\Sigma_{1}$ measures the response due to application of $\nabla \mu_{1}$ on the non-Lifshitz matter. The application of this gradient is what allows the conductivities to be finite even in the absence of momentum dissipation, i.e., $k \rightarrow 0$. This is an expected feature in a system with two species of $U(1)$ fields, first observed by Sonner [41] and later by Cremonini and Pope [31]. Our conductivities calculated through linear time sources are, of course, identical to the Neumann conductivities found by Cremonini, Cvetič and Papadimitriou [32]. This conductivity feature is an instance of some of the more robust behavior a $U(1) \times U(1)$ model can afford.

The continuity equation (39) in concert with the conductivities (42) and susceptibilities (42) yields a diffusion equation for energy and charge:

$$
\left(\begin{array}{c}
\partial_{t} \rho_{2} \\
\partial_{t} \epsilon
\end{array}\right)=\boldsymbol{D}\left(\begin{array}{c}
\nabla^{2} \rho_{2} \\
\nabla^{2} \epsilon
\end{array}\right)
$$

where the diffusion matrix $\boldsymbol{D}$ is determined from the conductivity matrix $\sigma$ and susceptibility matrix $\chi$ via the celebrated Einstein relation,

$$
D=\sigma \chi^{-1} .
$$

The diffusion eigenvalues follow

$$
\begin{aligned}
D_{+} D_{-} & =\frac{\kappa}{c_{\rho_{2}}} \frac{\sigma}{\chi} \\
D_{+}+D_{-} & =\frac{\kappa}{c_{\rho_{2}}}+\frac{\sigma}{\chi}+\frac{T \sigma}{c_{\rho_{2}}}\left(\frac{\zeta}{\chi}-\frac{\alpha}{\sigma}\right)^{2},
\end{aligned}
$$

where we define

$$
c_{\rho_{2}}=c_{\mu_{2}}-\frac{T \zeta^{2}}{\chi}
$$


as the specific heat for fixed electric charge — which follows from Maxwell relations-and

$$
\kappa=\bar{\kappa}-\frac{T \alpha^{2}}{\sigma},=\frac{4 \pi s T}{\Sigma_{1} k^{2} X\left(\phi_{+}\right)+\frac{4 \pi \rho_{2}^{2}}{r_{+}^{d_{s}-2} Z_{2}\left(\phi_{+}\right) s}}
$$

to be the open-circuit thermal conductivity, where no electric charge can flow. We note that $\kappa$ is explicitly dependent on both the metric and $A_{1}$, in contrast to traditional holographic systems where $\kappa$ is explicitly dependent only upon the form of the metric [18]. This is a direct consequence of our fixed Lifshitz charge scenario.

We will measure the diffusion eigenvalues relative to the butterfly velocity,

$$
v_{B}^{2}=\frac{2 \pi}{d_{s}} \operatorname{Tr}_{+}^{z-2},
$$

which will make $D_{ \pm} T / v_{B}^{2}$ pure numbers. This velocity is proposed to be the characteristic velocity for Lifshitz geometries and is independent of all matter content [17]. Rescaling Eq. (12) yields

$$
\begin{aligned}
& 1=\frac{R^{z}}{4 \pi}\left(z+d_{s}-\frac{\left(z+d_{s}-2\right)^{2}}{2 d_{s}} \tilde{\mu}_{2}^{2} R^{-2}-\frac{1}{2} \tilde{k}^{2} R^{-2 z}\right), \quad(50) \\
& r_{+}=T^{1 / z} R\left(\tilde{\mu}_{2}, \tilde{k}\right), \quad \tilde{\mu}_{2}=\frac{\mu_{2} e^{(z-1) \phi_{1}}}{T}, \quad \tilde{k}=\frac{k e^{-(z-1) \phi_{1}}}{T^{z}},
\end{aligned}
$$

and all pure numbers in our system will be functions of the two parameters $\tilde{\mu}_{2}$ and $\tilde{k}$. Written in scaling form, the determinant and trace of the diffusion matrix are given, respectively, by

$$
\begin{aligned}
& \frac{D_{+} D_{-} T^{2}}{v_{B}^{4}} \\
& =\frac{z d_{s}\left[2\left(z+d_{s}\right)+\tilde{k}^{2} R^{-2 z}\right]-(z-2)\left(z+d_{s}-2\right)^{2} \tilde{\mu}^{2} R^{-2}}{8 \pi^{2}\left(z+d_{s}-2\right)\left[2(z-1)\left(z+d_{s}\right)+\tilde{k}^{2} R^{-2 z}\right]},
\end{aligned}
$$

$$
\begin{aligned}
& \frac{\left(D_{+}+D_{-}\right) T}{v_{B}^{2}} \\
& =\frac{d_{s}}{2 \pi\left(z+d_{s}-2\right)} \\
& +\frac{z d_{s}\left[2\left(z+d_{s}\right)+\tilde{k}^{2} R^{-2 z}\right]+(z-2)\left[(z-2)^{2}-d_{s}^{2}\right] \tilde{\mu}_{2}^{2} R^{-2}}{4 \pi d_{s}\left[2(z-1)\left(z+d_{s}\right)+\tilde{k}^{2} R^{-2 z}\right]} .
\end{aligned}
$$

We will always order the eigenvalues such that $D_{+} \geq D_{-}$. We display a couple of solutions explicitly in Figs. 2 and 3. Notably, we find the diffusion eigenvalues are
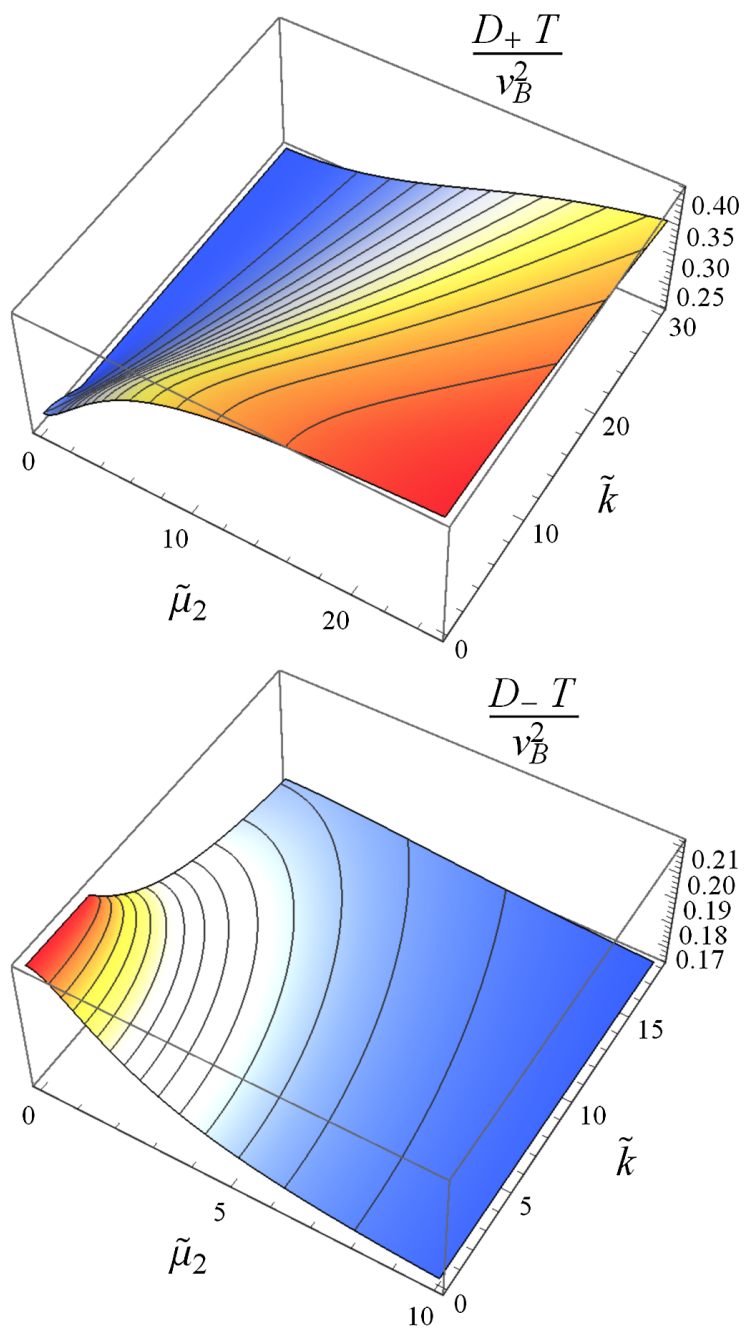

FIG. 2. $D_{ \pm} T / v_{B}^{2}$ for $d_{s}=2$ and $z=3 / 2$.

bounded. The bounds can be obtained analytically through various limits of $\tilde{\mu}_{2}$ and $\tilde{k}$ and are given by

$$
\begin{aligned}
& \max \left(\frac{D_{+} T}{v_{B}^{2}}\right)=\left\{\begin{array}{ll}
\frac{d_{s}}{2 \pi(z-1)\left(z+d_{s}-2\right)} & z<2, \\
\frac{1}{2 \pi} & z \geq 2
\end{array},\right. \\
& \min \left(\frac{D_{+} T}{v_{B}^{2}}\right)=\left\{\begin{array}{ll}
\frac{z}{4 \pi(z-1)} & z>2 \text { and } z>d_{s} \\
\frac{d_{s}}{2 \pi\left(z+d_{s}-2\right)} & \text { else }
\end{array},\right. \\
& \max \left(\frac{D_{-} T}{v_{B}^{2}}\right)= \begin{cases}\frac{z}{4 \pi(z-1)} & d_{s}<z<2, \\
\frac{d_{s}}{2 \pi\left(z+d_{s}-2\right)} & \text { else }\end{cases}
\end{aligned}
$$

$$
\min \left(\frac{D_{-} T}{v_{B}^{2}}\right)=\left\{\begin{array}{ll}
\frac{1}{2 \pi} & z \leq 2 \\
\frac{d_{s}}{2 \pi(z-1)\left(z+d_{s}-2\right)} & z>2
\end{array},\right.
$$



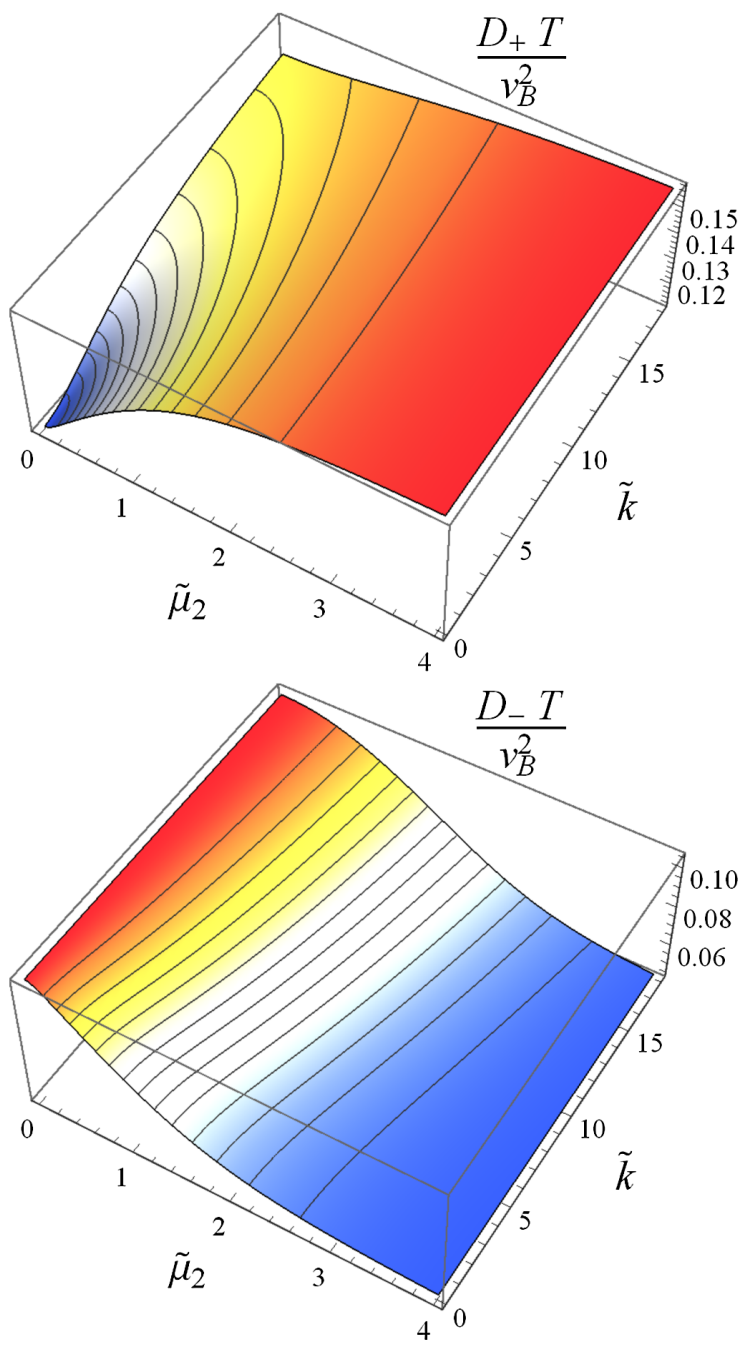

FIG. 3. $D_{ \pm} T / v_{B}^{2}$ for $d_{s}=3$ and $z=7 / 2$.

which are shown in Fig. 4 for $d_{s}=\{1,2,3\}$, in which the primacy of $z=2$ as the scale-invariant point of the diffusion equation is evident. To illustrate this further, we plot Eq. (3) with the length defined as $\ell=2 \pi / \tilde{k}$ in Fig. 5. ${ }^{1}$ This characteristic length functions as an effective lattice spacing. As is evident, there is a universal sign change at $z=2$. At $z=2$, the diffusion constants are equal and given by the universal value,

$$
D_{ \pm}=\frac{1}{d_{s}}
$$

as remarked in the Introduction. The universal nature of the charge and energy diffusion constants stems from the underlying scale invariance of the diffusion equation when $z=2$. The sign change of $\beta$ signals a fixed point exists at

\footnotetext{
${ }^{1}$ We could very well have used $\operatorname{det} \boldsymbol{D}$ instead of $\operatorname{tr} \boldsymbol{D}$, though the qualitative features are identical.
}
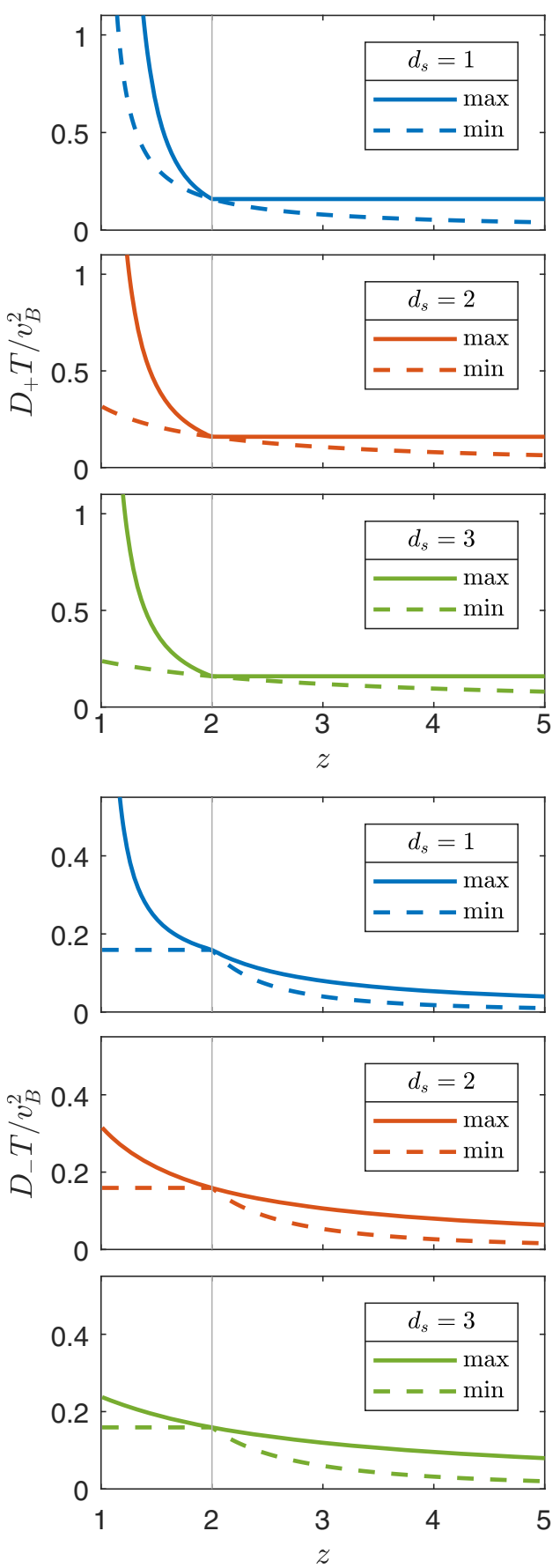

FIG. 4. The bounds of $D_{ \pm} T / v_{B}^{2}$ for $d_{s}=\{1,2,3\}$. The minimum of $D_{+} T / v_{B}^{2}$ asymptotes to $1 / 4 \pi$ as $z \rightarrow \infty$.

$z=2$ analogous to the scale invariance that obtains for the conductance in the case of $d_{s}=2$ in Anderson localization [19]. Necessarily, the diffusion scale $v_{B}^{2} / T$ is also a pure number at this point, as seen in Eq. (49) where the horizon dependence vanishes. The marginality of $z=2$ has been noted in other contexts such as a Lifshitz string [42] and the stability of scalar hair [43]. In particular, the so-called perfect fluid, which depends only upon its own rest mass and isotropic pressure, can only exist in concert with 


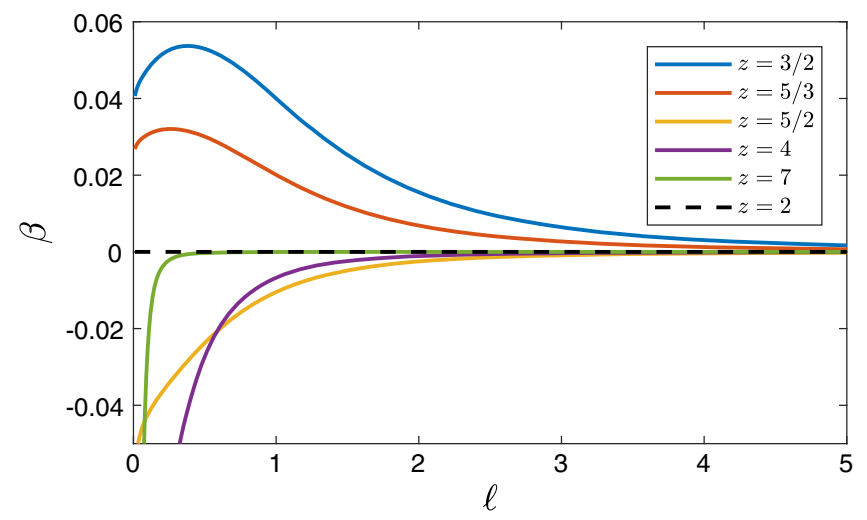

FIG. 5. Plot of the $\beta$ function [Eq. (3)] illustrating the universality of the diffusivities as a function of length for varying $z$, using $\tilde{\mu}_{2}=1.8$ and $d_{s}=3$. The qualitative features of $\beta$ are independent of the chemical potential and the number of spatial dimensions. The universal sign change signifies that the length dependence of the diffusivities is controlled by a fixed point at $z=2$.

Galilean boosts at $z=2$ wherein the scale-dependent mass contribution drops out [44]. Note that we have allowed noninteger values of $d_{s}$ in our solutions, which can effectively be obtained through the use of a hyperscaling violating parameter.

To put our results for the transport in the context of expected results, we first observe the universal features. In the decoupling limit where the chemical potential is turned off, we find the charge and energy diffusion constants follow

$$
\begin{aligned}
\lim _{\tilde{\mu}_{2} \rightarrow 0} \frac{\sigma}{\chi} \frac{T}{v_{B}^{2}} & =\frac{d_{s}}{2 \pi\left(z+d_{s}-2\right)}, \\
\lim _{\tilde{\mu}_{2}, \tilde{k} \rightarrow 0} \frac{\kappa}{c_{\rho_{2}}} \frac{T}{v_{B}^{2}} & =\frac{z}{4 \pi(z-1)},
\end{aligned}
$$

which exactly match the purported decoupled forms in $[17,18]$. For $z \geq 2$ and $z \geq d_{s}$, which heads toward the decoupling limit of large $z$, we find these forms match $\max \left(D_{-} T / v_{B}^{2}\right)$ and $\min \left(D_{+} T / v_{B}^{2}\right)$ exactly. The limits in which deviations occur are laid bare in Fig. 3. For $D_{-}$, deviation from this behavior is found when $\tilde{\mu}_{2} \gg 1$, the limit in which we expect thermoelectric interactions to be quite strong and so inhibit charge flow. For $D_{+}$, deviation is found under either the conditions $\tilde{k} \gg 1$ or $\tilde{\mu}_{2} \gg 1$, emphasizing that energy diffusivity is heavily subject to all matter interactions.

A limit of interest is $z \rightarrow \infty$. In particular, the bounds indicate clearly that $D_{-} T / v_{B}^{2} \rightarrow 0$, signaling that charge does not diffuse in this limit, only energy. Conventionally, $z \rightarrow \infty$ corresponds to localized critical physics; the divergence of the critical length guarantees no dynamic critical behavior can obtain on any appreciable time scale. For our system, we can interpret this to mean charge must follow this type of quantum critical behavior but energy does not. We also notice the saturation of the $D_{+}$diffusion constant for $z \geq 2$, whereupon $1 / 4 \pi \leq D_{+} T / v_{B}^{2} \leq 1 / 2 \pi$. The upper bound is the typical saturation observed in the SYK model [45]. Intuitively, this characterizes the fact that energy diffusivity must be bounded from below and above, so long as momentum dissipation is present, regardless of the value of $z$.

\section{CONCLUSIONS}

From this detailed treatment of Lifshitz holography, we have been able to derive a series of thermodynamic and dynamical response functions. Of particular note is the explicit derivation of both the Bekenstein-Hawking and Smarr-like relationships, made possible by the exact computation of the renormalized thermodynamic potential. Our calculations reveal the universal features of the diffusion constants near $z=2$ even in the complicated setting in which charge and thermal degrees of freedom are treated on equal footing. This universality obtains because of the emergence of a fixed point characterizing the length dependence of the diffusivities at $z=2$. The vanishing of the charge diffusion constant in the local critical limit of $z \rightarrow \infty$ represents the ultimate deviation from the expected bounds. Since our treatment fully incorporates thermodynamics and electrical responses, it should serve as a template for extracting the coterie of transport coefficients relevant to quantum critical matter.

In this work, thermodynamic quantities were computed explicitly working with a derived free energy expression. We have considered here only diffusion in the context of a fixed Lifshitz field. It is perhaps possible to relax the Lifshitz source constraint in this methodology by introducing a double-trace deformation for $A_{1}$. Such a parameter generally would have distinct scaling from any $J_{1}^{2}$ counterterm already present, and would incorporate a completely free coefficient to utilize as an independent source, thus enabling a treatment in the Dirichlet scheme. The various response functions can be computed through careful and copious Maxwell relations.

\section{ACKNOWLEDGMENTS}

We would like to thank Matteo Baggioli, Garrett Vanacore, Tom Faulkner, Rob Leigh, Michael Blake, Sean Hartnoll and Julian Sonner for comments and discussion along the way. We also thank the NSF DMR1461952 and DMR-1919143 for partial funding of this project.

\section{APPENDIX A: FULL BACKGROUND RENORMALIZATION}

For $k \neq 0$, we can have a series of divergent terms in Eq. (14). Consider counterterms 


$$
I_{\text {c.t. }}=\int_{\partial \mathcal{M}} \sum_{n=0}\left(c_{n}^{(\mathrm{vol})} \sqrt{-\gamma}+c_{n}^{\left(J_{1}^{2}\right)} \frac{J_{1}^{2}}{\sqrt{-\gamma} Z_{1}(\phi)}\right)\left[X(\phi) \sum_{I=1}^{d_{s}}\left(\hat{\nabla} \chi_{I}\right)^{2}\right]^{n},
$$

whose contributions to our boundary responses are

$$
\begin{gathered}
\mathbb{T}_{b \mid c . t .}^{a}=\sum_{n=0}\left[X(\phi) \sum_{J=1}^{d_{s}}\left(\hat{\nabla} \chi_{J}\right)^{2}\right]^{n-1} 2 X(\phi) \sum_{I=1}^{d_{s}}\left[c_{n}^{(\mathrm{vol})} \sqrt{-\gamma}\left(\frac{1}{2} \delta^{a}{ }_{b}\left(\hat{\nabla} \chi_{I}\right)^{2}-n \hat{\nabla}^{a} \chi_{I} \hat{\nabla}_{b} \chi_{I}\right)\right. \\
\left.+\frac{c_{n}^{\left(J_{1}^{2}\right)}}{\sqrt{-\gamma} Z_{1}(\phi)}\left(J_{1}^{a} J_{1, b}\left(\hat{\nabla} \chi_{I}\right)^{2}-n J_{1}^{2} \hat{\nabla}^{a} \chi_{I} \hat{\nabla}_{b} \chi_{I}-\frac{1}{2} \delta_{b}^{a} J_{1}^{2}\right)\left(\hat{\nabla} \chi_{I}\right)^{2}\right] \\
\mathbb{A}_{1, a \mid c . t .}=\sum_{n=0}\left[X(\phi) \sum_{I=1}^{d_{s}}\left(\hat{\nabla} \chi_{I}\right)^{2}\right]^{n} \frac{2 c_{n}^{\left(J_{1}^{2}\right)} J_{1, a}}{\sqrt{-\gamma} Z_{1}(\phi)}, \\
\mathbb{O}_{\phi \mid c . t .}=\sum_{n=0}\left[X(\phi) \sum_{I=1}^{d_{s}}\left(\hat{\nabla} \chi_{I}\right)^{2}\right]^{n}\left[c_{n}^{(\mathrm{vol})} \sqrt{-\gamma} \frac{n X^{\prime}(\phi)}{X(\phi)}+c_{n}^{\left(J_{1}^{2}\right)} \frac{J_{1}^{2}}{\sqrt{-\gamma} Z_{1}(\phi)}\left(\frac{n X^{\prime}(\phi)}{X(\phi)}-\frac{Z_{1}^{\prime}(\phi)}{Z_{1}(\phi)}\right)\right] .
\end{gathered}
$$

The covariant derivative $\hat{\nabla}_{a}$ is defined from the intrinsic metric $\gamma_{a b}$. In general, we can increment $n$ to cancel out higher order powers involving $k^{2}$ terms that bleed over from our established action. The constants are given by

$$
\begin{aligned}
& c_{n}^{(\mathrm{vol})}=\vartheta_{n}^{(\mathrm{vol})} \frac{(2 n-1)(z-1)-2 d_{s}}{\left[4 d_{s}\left(d_{s}-z\right)\right]^{n}}, \\
& c_{n}^{\left(J_{1}^{2}\right)}=\vartheta_{n}^{\left(J_{1}^{2}\right)} \frac{1}{\left(z+d_{s}\right)\left[4 d_{s}\left(d_{s}-z\right)\right]^{n}},
\end{aligned}
$$

and are determined recursively from $n=0$ with $\vartheta_{n}^{(\mathrm{vol})}=$ $\left\{-1,1, \frac{1}{2}, \frac{1}{2}, \frac{5}{8}, \frac{7}{8}, \ldots\right\}$ and $\vartheta_{n}^{\left(J_{1}^{2}\right)}=\left\{\frac{1}{2}, \frac{1}{2}, \frac{3}{4}, \frac{5}{4}, \frac{35}{16}, \frac{63}{16}, \ldots\right\}$. This is a specific encoding of the renormalization provided by the Hamilton-Jacobi equations, which expands in a series of functional derivatives [36,37].

We would like to emphasize that the counterterm series equation (A1) should terminate finitely in conjunction with finite $z$ and $d_{s}$ as each term in the series is increasingly subleading. Hence, the counterterms are not only finitely numerable but also unique.

\section{APPENDIX B: KILLING VECTOR CONSERVED QUANTITY}

Here we determine the bulk conserved quantity that will be dual to the heat current as outlined in [46]. Suppose there exists a Killing vector $\xi$, defined by

$$
\mathcal{L}_{\xi} g_{a b}=\nabla_{(a} \xi_{b)}=0,
$$

which of course can correspond to an infinitesimal diffeomorphism. We consider that the Lie derivatives on our physical observable fields vanishes; that is

$$
\mathcal{L}_{\xi} F_{q, a b}=\mathcal{L}_{\xi} \phi=\mathcal{L}_{\xi} \chi_{I}=0
$$

The first of these, rewritten, states that

$$
\left(i_{\xi} d+d i_{\xi}\right) F_{q}=0
$$

and thus we can assume that $i_{\xi} F_{q}$ is an exact form

$$
i_{\xi} F_{q}=d \theta_{q}
$$

for some functions $\theta_{q}$. This also implies that we can express

$$
\mathcal{L}_{\xi} A_{q}=d \psi_{q}
$$

for some functions $\psi_{q}$. These identities will allow us to construct a total derivative by examining

$$
\begin{aligned}
\nabla_{b} \nabla^{a} \xi^{b}= & R_{b}^{a} \xi^{b} \\
= & \frac{1}{d_{s}} V(\phi) \xi^{a} \\
& +\frac{1}{2} \sum_{q=1}^{2}\left(\xi^{c} Z_{q}(\phi) F_{q}^{a b} F_{q, c b}-\frac{1}{2 d_{s}} \xi^{a} Z_{q}(\phi) F_{q}^{2}\right) .
\end{aligned}
$$

This expression can be rearranged as

$$
\nabla_{b} G^{a b}=\frac{1}{d_{s}} V(\phi) \xi^{a},
$$

where 


$$
\begin{aligned}
G^{a b}= & \nabla^{a} \xi^{b} \\
& +\frac{1}{2 d_{s}} \sum_{q=1}^{2} Z_{q}(\phi)\left[\left(\psi_{q}-d_{s} \theta_{q}\right) F_{q}^{a b}+2 \xi^{[a} F_{q}^{b] c} A_{q, c}\right] .
\end{aligned}
$$

To deduce this expression, we use the identities

$$
\begin{gathered}
\xi^{c} Z_{q}(\phi) F_{q}^{a b} F_{q, c b}=\nabla_{b}\left(\theta_{q} Z_{q}(\phi) F_{q}^{a b}\right), \\
\xi^{a} Z_{q}(\phi) F_{q}^{2}=\nabla_{b}\left(4 \xi^{[a} Z_{q}(\phi) F_{q}^{b] c} A_{q, c}+2 \psi_{q} Z_{q}(\phi) F_{q}^{a b}\right),
\end{gathered}
$$

where the latter of these can be realized from rearranging the Lie derivative $\mathcal{L}_{\xi} F_{q, a b}=\xi^{c} \nabla_{c} F_{q, a b}+F_{q, b c} \nabla_{a} \xi^{c}+$ $F_{q, a c} \nabla_{b} \xi^{c}=0$. Now, as long as any components of $\xi$ vanish we can deduce a conserved quantity. By choosing $\xi=\nabla_{t}$, it is clear that the $x^{i}$ components then generate conserved quantities, which are dual to the boundary heat currents.

\section{APPENDIX C: RENORMALIZATION FOR CURRENTS}

With the counterterms provided in Eq. (16), we find that

$$
\mathbb{T}^{x^{1}}=j^{(\epsilon)}
$$

and as such we would like to construct our boundary theory such that this is the response to our metric variation. Presently, we would find that the boundary variation leaves a source term [46] in the action. This is not a problem for $z=1$, but otherwise this term is divergent. To mollify this divergence, we can recast the intrinsic metric $\gamma_{a b}$ as

$$
\gamma_{a b} d x^{a} d x^{b}=-n^{2}\left(d t-\frac{n_{i}}{n^{2}} d x^{i}\right)^{2}+\sigma_{i j} d x^{i} d x^{j},
$$

akin to the ADM formalism but where our boundary spacetime is instead foliated by the normalized timelike covector $\frac{1}{n} \nabla_{t}$. Then we can consider the fundamental variational objects of our theory to be $n, n_{i}$ and $\sigma_{i j}$ instead of the boundary metric. Our variation becomes

$$
\begin{aligned}
\delta I= & \int_{\partial \mathcal{M}}\left[\left(-\mathbb{t}^{t t} n^{2}+\mathbb{T}^{i j} \frac{n_{i} n_{j}}{n^{2}}\right) \frac{\delta n}{n}\right. \\
& \left.-\mathbb{T}^{i}{ }_{t} \frac{\delta n_{i}}{n^{2}}+\frac{1}{2} \mathbb{T}^{i j} \delta \sigma_{i j}+\cdots\right],
\end{aligned}
$$

and now the source term present in the $\delta n_{i}$ term decays. Additionally, thanks to our formulation of $n_{i}$ as a small parameter, the response to the normalized variation of the lapse $\delta n / n$ is exactly $\mathbb{T}^{t}$. Thus nothing about our static background scheme is modified.

\section{APPENDIX D: LINEAR TIME SOURCES}

Linear time sources provide a straightforward scheme for deducing DC response functions. Additionally, through our gauge symmetries, they have a clear interpretation as temperature and chemical potential gradients. We can transform the metric and $U(1)$ fields as

$$
\begin{aligned}
g_{a b} & \rightarrow g_{a b}+\mathcal{L}_{\xi} g_{a b}, \\
A_{q, a} & \rightarrow A_{q, a}+\mathcal{L}_{\xi} A_{q, a}+\nabla_{a} \Lambda_{q},
\end{aligned}
$$

where $\xi$ parametrizes an infinitesimal coordinate transformation $x^{a} \rightarrow x^{a}+\xi^{a}$ and each $\Lambda_{q}$ a $U(1)$ transformation, respectively. For the choices

$$
\begin{aligned}
\xi & =-t x^{1} \frac{\nabla T}{T} \nabla_{t}, \\
\Lambda_{q} & =t x^{1} \nabla \mu_{q},
\end{aligned}
$$

our ansatz sources become

$$
\begin{aligned}
\delta d s^{2} & =2 r^{2 z} f x^{1} \frac{\nabla T}{T} d t^{2}, \\
\delta A_{q} & =x^{1}\left(\nabla \mu_{q}-A_{q, t} \frac{\nabla T}{T}\right) d t,
\end{aligned}
$$

which are exactly the gradients we would expect for small perturbations of $T$ and $\mu_{q}$. The form of the temperature fluctuations are determined by perturbing the period of Euclidean time, $1 / T$.
[1] A. Bianconi, Lifshitz transitions in multi-band hubbard models for topological superconductivity in complex quantum matter, J. Supercond. Novel Magn. 31, 603 (2018).

[2] M. R. Norman, J. Lin, and A. J. Millis, Lifshitz transition in underdoped cuprates, Phys. Rev. B 81, 180513 (2010).
[3] X. Shi, Z.-Q. Han, X.-L. Peng, P. Richard, T. Qian, X.-X. Wu et al., Enhanced superconductivity accompanying a Lifshitz transition in electron-doped fese monolayer, Nat. Commun. 8, 14988 (2017).

[4] G. E. Volovik, Topological Lifshitz transitions, Low Temp. Phys. 43, 47 (2017). 
[5] S. Kachru, X. Liu, and M. Mulligan, Gravity duals of Lifshitz-like fixed points, Phys. Rev. D 78, 106005 (2008).

[6] P. Hořava, Quantum gravity at a Lifshitz point, Phys. Rev. D 79, 084008 (2009).

[7] K. Balasubramanian and J. McGreevy, The particle number in Galilean holography, J. High Energy Phys. 01 (2011) 137.

[8] E. Kiritsis, Lorentz violation, gravity, dissipation and holography, J. High Energy Phys. 01 (2013) 030.

[9] K. Jensen, On the coupling of Galilean-invariant field theories to curved spacetime, SciPost Phys. 5, 11 (2018).

[10] B. Goutéraux and E. Kiritsis, Generalized holographic quantum criticality at finite density, J. High Energy Phys. 12 (2011) 036.

[11] Z. Fan, Holographic superconductors with hyperscaling violation, J. High Energy Phys. 09 (2013) 048.

[12] B. Goutéraux, Charge transport in holography with momentum dissipation, J. High Energy Phys. 04 (2014) 181.

[13] J. Tarrio, Asymptotically Lifshitz black holes in EinsteinMaxwell-dilaton theories, Fortschr. Phys. 60, 1098 (2012).

[14] E. Kiritsis and Y. Matsuo, Charge-hyperscaling violating Lifshitz hydrodynamics from black-holes, J. High Energy Phys. 12 (2015) 001.

[15] E. Kiritsis and Y. Matsuo, Hyperscaling-violating Lifshitz hydrodynamics from black-holes: Part II, J. High Energy Phys. 03 (2017) 041.

[16] I. Papadimitriou, Hyperscaling violating Lifshitz holography, Nucl. Part. Phys. Proc. 273-275, 1487 (2016).

[17] M. Blake, Universal Charge Diffusion and the Butterfly Effect in Holographic Theories, Phys. Rev. Lett. 117, 091601 (2016).

[18] M. Blake, R. A. Davison, and S. Sachdev, Thermal diffusivity and chaos in metals without quasiparticles, Phys. Rev. D 96, 106008 (2017).

[19] E. Abrahams, P. W. Anderson, D. C. Licciardello, and T. V. Ramakrishnan, Scaling Theory of Localization: Absence of Quantum Diffusion in Two Dimensions, Phys. Rev. Lett. 42, 673 (1979).

[20] D.-W. Pang, Conductivity and diffusion constant in Lifshitz backgrounds, J. High Energy Phys. 01 (2010) 120.

[21] X.-H. Ge, S.-J. Sin, Y. Tian, S.-F. Wu, and S.-Y. Wu, Charged BTZ-like black hole solutions and the diffusivitybutterfly velocity relation, J. High Energy Phys. 01 (2018) 068.

[22] S. A. Hartnoll, Theory of universal incoherent metallic transport, Nat. Phys. 11, 54 (2015).

[23] T. Hartman, S. A. Hartnoll, and R. Mahajan, Upper Bound on Diffusivity, Phys. Rev. Lett. 119, 141601 (2017).

[24] D. Cassani and A. F. Faedo, Constructing Lifshitz solutions from AdS, J. High Energy Phys. 05 (2011) 013.

[25] T. Andrade and S. F. Ross, Boundary conditions for metric fluctuations in Lifshitz, Classical Quantum Gravity 30, 195017 (2013).

[26] I. Papadimitriou, Hyperscaling violating Lifshitz holography, Nucl. Part. Phys. Proc. 273-275, 1487 (2016).

[27] V. Balasubramanian and P. Kraus, A stress tensor for antide Sitter gravity, Commun. Math. Phys. 208, 413 (1999).
[28] W. Chemissany, D. Geissbühler, J. Hartong, and B. Rollier, Holographic renormalization for $z=2$ Lifshitz spacetimes from AdS, Classical Quantum Gravity 29, 235017 (2012).

[29] V. Keränen and L. Thorlacius, Thermal correlators in holographic models with Lifshitz scaling, Classical Quantum Gravity 29, 194009 (2012).

[30] M. Taylor, Lifshitz holography, Classical Quantum Gravity 33, 033001 (2016).

[31] S. Cremonini, H.-S. Liu, H. Lü, and C. Pope, DC conductivities from non-relativistic scaling geometries with momentum dissipation, J. High Energy Phys. 04 (2017) 009.

[32] S. Cremonini, M. Cvetič, and I. Papadimitriou, Thermoelectric DC conductivities in hyperscaling violating Lifshitz theories, J. High Energy Phys. 04 (2018) 099.

[33] M. Alishahiha, E. Ó. Colgáin, and H. Yavartanoo, Charged black branes with hyperscaling violating factor, J. High Energy Phys. 11 (2012) 137.

[34] X.-M. Kuang and J.-P. Wu, Analytical shear viscosity in hyperscaling violating black brane, Phys. Lett. B 773, 422 (2017).

[35] B. C. van Rees, Holographic renormalization for irrelevant operators and multi-trace counterterms, J. High Energy Phys. 08 (2011) 093.

[36] I. Papadimitriou, Holographic renormalization of general dilaton-axion gravity, J. High Energy Phys. 08 (2011) 119.

[37] W. Chemissany and I. Papadimitriou, Lifshitz holography: The whole shebang, J. High Energy Phys. 01 (2015) 052 .

[38] I. Arav, Y. Oz, and A. Raviv-Moshe, Lifshitz anomalies, Ward identities and split dimensional regularization, J. High Energy Phys. 03 (2017) 088.

[39] J. Hartong, E. Kiritsis, and N. A. Obers, Field theory on Newton-Cartan backgrounds and symmetries of the Lifshitz vacuum, J. High Energy Phys. 08 (2015) 006.

[40] T. Andrade and B. Withers, A simple holographic model of momentum relaxation, J. High Energy Phys. 05 (2014) 101.

[41] J. Sonner, On universality of charge transport in AdS/CFT, J. High Energy Phys. 07 (2013) 145.

[42] D. Tong and K. Wong, Fluctuation and Dissipation at a Quantum Critical Point, Phys. Rev. Lett. 110, 061602 (2013).

[43] M. R. M. Mozaffar and A. Mollabashi, Quantum critical points in Lifshitz space-time, J. High Energy Phys. 04 (2013) 081.

[44] J. de Boer, J. Hartong, N. A. Obers, W. Sybesma, and S. Vandoren, Perfect fluids, SciPost Phys. 5, 3 (2018).

[45] R. A. Davison, W. Fu, A. Georges, Y. Gu, K. Jensen, and S. Sachdev, Thermoelectric transport in disordered metals without quasiparticles: The Sachdev-Ye-Kitaev models and holography, Phys. Rev. B 95, 155131 (2017).

[46] A. Donos and J. P. Gauntlett, Thermoelectric DC conductivities from black hole horizons, J. High Energy Phys. 11 (2014) 081. 